Provided for non-commercial research and educational use. Not for reproduction, distribution or commercial use.

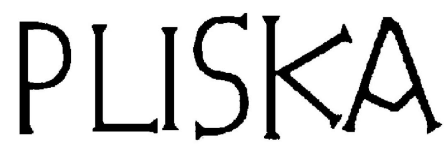

\title{
STUDIA MATHEMATICA
}

BULGARICA

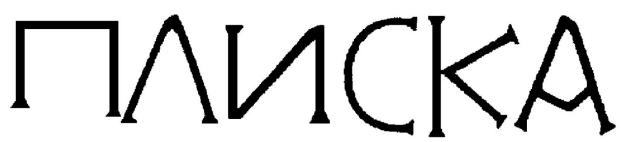

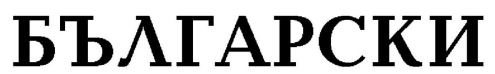

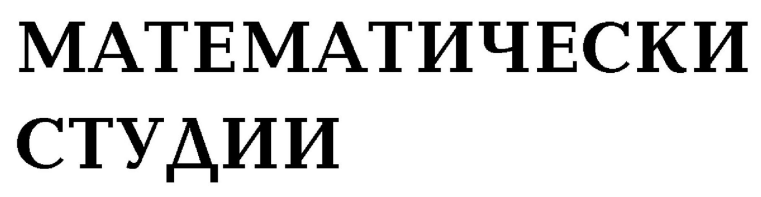

The attached copy is furnished for non-commercial research and education use only.

Authors are permitted to post this version of the article to their personal websites or institutional repositories and to share with other researchers in the form of electronic reprints.

Other uses, including reproduction and distribution, or selling or licensing copies, or posting to third party websites are prohibited.

For further information on

Pliska Studia Mathematica Bulgarica

visit the website of the journal http://www.math.bas.bg/ pliska/

or contact: Editorial Office

Pliska Studia Mathematica Bulgarica

Institute of Mathematics and Informatics

Bulgarian Academy of Sciences

Telephone: (+359-2)9792818, FAX:(+359-2)971-36-49

e-mail: pliska@math.bas.bg 


\title{
ON SELF-SIMILAR EXTREMAL PROCESSES*
}

\author{
Elisaveta I. Pancheva
}

Given an extremal process $X:[0, \infty) \rightarrow[0, \infty)^{d}$ with lower curve $C$ and associated point process $N=\left\{\left(t_{k}, X_{k}\right): k \geq 0\right\}, t_{k}$ distinct and $X_{k}$ independent, given a sequence $\zeta_{n}=\left(\tau_{n}, \xi_{n}\right), n \geq 1$ of time-space changes (max-automorphisms of $\left.[0, \infty)^{d+1}\right)$, we study the limit behaviour of the sequence of extremal processes

$$
\begin{aligned}
Y_{n}(t) & =\xi_{n}^{-1} \circ X \circ \tau_{n}(t) \\
& =C_{n}(t) \vee \max \left\{\xi_{n}^{-1} \circ X_{k}: t_{k} \leq \tau_{n}(t)\right\} \Longrightarrow Y
\end{aligned}
$$

under a regularity condition on the norming sequence $\zeta_{n}$ and asymptotic negligibility of the max-increments of $Y_{n}$. The limit class consists of self-similar (w.r.t. a group $\eta_{\alpha}=\left(\sigma_{\alpha}, L_{\alpha}\right), \alpha>0$, of time-space changes) extremal processes. Under self-similarity here we understand the property $L_{\alpha} \circ Y(t) \stackrel{d}{=} Y \circ \sigma_{\alpha}(t)$, for all $\alpha>0$. The univariate marginals of $Y$ are max-selfdecomposable. If additionally the initial extremal process $X$ is supposed to have homogeneous max-increments then the limit process is max-stable with homogeneous max-increments.

Keywords: multivariate extremal processes ; self-similarity; homogeneous max-increments; weak convergence.

\section{Introduction}

An extremal process $Y:[0, \infty) \rightarrow[0, \infty)^{d}$ is a stochastic process with the following two properties :

1) The sample paths are right continuous increasing functions from the half line $[0, \infty)$ to the positive orthand $[0, \infty)^{d}$, called time space and state space, respectively.

2) For any finite sequence of time points $0=t_{0}<\ldots<t_{m}$ there exist independent rv's $U_{0}, \ldots, U_{m}$ in $[0, \infty)^{d}$ such that

$$
\left(Y\left(t_{0}\right), \ldots, Y\left(t_{m}\right)\right) \stackrel{d}{=}\left(U_{0}, U_{0} \vee U_{1}, \ldots, U_{0} \vee \ldots \vee U_{m}\right)
$$

${ }^{*}$ This paper is partially supported by the Bulgarian Ministry of Education and Sciences under grand MM 234/1996 
The probability distribution of an extremal process with independent max-increments $U$ is completely determined by its distribution function (df). The df of an extremal process $Y$ is the function $f:(0, \infty)^{d+1} \rightarrow[0,1]$,

$$
f(t, x)=P(Y(t)<x) .
$$

It is decreasing and right continuous in $t$ and increasing and left continuous in $x$, so lower semicontinuous.

With an extremal process $Y$ we associate a lower curve $C_{Y}:[0, \infty) \rightarrow[0, \infty)^{d}$, increasing and right continuous, below which the sample functions of $Y$ can not pass. It is defined coordinatewise: $C^{(i)}(t)$ is the lower endpoint of the $\mathrm{df} F_{t}^{(i)}$ of the ith coordinate of the $\mathrm{rv} Y(t), i=1 \ldots d$. Any extremal process determines uniquely its lower curve. in $[4]$.

The following two fundamental results for multivariate extremal processes are stated

Theorem 1 Structure theorem. Let $Y:[0, \infty) \rightarrow[0, \infty)^{d}$ be an extremal process with lower curve $C$. If the underlying probability space is sufficiently rich, there exists a consistent family of max-increments $U(s, t), 0 \leq s<t$, such that

1) $U(s, t) \geq C(t)$ a.s.,$s<t$;

2) $Y(t)=Y(s) \vee U(s, t)$ a.s.,$s<t$;

3) for any finite sequence of time points $0=t_{0}<\ldots<t_{m}$ the $m+1$ vectors $Y(0), U\left(t_{0}, t_{1}\right), \ldots, U\left(t_{m-1}, t_{m}\right)$ are independent.

So, an extremal process is uniquely determined by a given family of max-increments. The converse is not always true: Different families of max-increments may lead to the same extremal process. This phenomenon, called blotting, is studied in [4].

Theorem 2 Decomposition theorem. Let $Y:[0, \infty) \rightarrow[0, \infty)^{d}$ be an extremal process with lower curve $C$ and a consistent family of max-increments. Then $Y$ is the maximum of two independent extremal processes $Y^{\prime}$ and $Y^{\prime \prime}$ with common lower curve $C$. The process $Y^{\prime}$ is generated by a Poisson point process $N^{\prime}$ which mean measure does not charge any instant space $S_{t}:=\{t\} \times[0, \infty)^{d}$. The associated with $Y$ " point process $N$ " is the sum of a sequence of independent $0-1$ point processes $N_{k}$ on $S_{t_{k}}$ and $t_{k}$, are distinct non-random time points. Both processes are independent.

Thus, if $\left(T_{k}, X_{k}\right), k \geq 1$ are points of the point process $N=N^{\prime}+N^{\prime \prime}$ then

$$
Y(t)=C_{Y}(t) \vee \max \left\{X_{k}: T_{k} \leq t\right\}
$$

and we say that the point process $N$ generates the extremal process $Y$. All realizations of the point process are supposed to be Radon measures on the open set $[0, C]^{c}=$ $\left([0, \infty) \times[0, \infty]^{d}\right) \backslash[0, C]$. Hence

$$
N\left([0, t] \times[0, x)^{c}\right)<\infty \quad \text { a.s. for } t \geq 0, x>C(t) .
$$


Let $\mathcal{M}_{d}$ be the set of all right continuous increasing functions $y:(0, \infty) \rightarrow[0, \infty)^{d}$. Denote convergence in law by $\Longrightarrow$. Then $Y_{n} \Longrightarrow Y_{0}$ weakly if the probability distributions $\pi_{n}$ of $Y_{n}$ converge :

$$
E \varphi\left(Y_{n}\right)=\int \varphi d \pi_{n} \rightarrow \int \varphi d \pi_{0}=E \varphi\left(Y_{0}\right)
$$

for each bounded function $\varphi: \mathcal{M}_{d} \rightarrow R$ which is continuous for the weak topology on $\mathcal{M}_{d}$.

If $\left(^{*}\right)$ holds for each bounded $\varphi: \mathcal{M}_{d} \rightarrow R$ which is continuous in the Skorohod topology $D([0, \infty))$, we write $Y_{N} \Longrightarrow Y_{0} \quad$ in $D([0, \infty))$.

Let $y_{n}:[0, \infty) \rightarrow[0, \infty)^{d}$ be right continuous and increasing for $n \geq 0$. Recall, the sequence $y_{n}$ converges to $y_{0}$ in $D([0, \infty))$ if:

i) $y_{n} \rightarrow y_{0}$ weakly on $(0, \infty)$;

ii) $y_{n}(0) \rightarrow y_{0}(0)$;

iii) for each discontinuity point $t>0$ of the function $y_{0}$ there is a sequence $t_{n} \rightarrow t$ so that

$$
y_{n}\left(t_{n}\right) \rightarrow y_{0}(t) \quad, \quad y_{n}\left(t_{n}-0\right) \rightarrow y_{0}(t-0) \quad .
$$

Consider arrays of the form $\left\{\left(t_{n k}, X_{n k}\right): k \geq 0\right\}, n \geq 1$, where $X_{n k}$ are row-wise independent rv's in $[0, \infty)^{d}$ and for each $n$ the sequence of deterministic time points $0=t_{n 0}<t_{n 1}<\ldots$ is strictly increasing to $\infty$. We transform an array into a sequence of extremal processes $Y_{n}$ with lower curves $C_{n}(t)$ by setting

$$
Y_{n}(t)=C_{n}(t) \vee \max \left\{X_{n k}: t_{n k} \leq t\right\} .
$$

By virtue of (2) the maximum of the RHS of (3) is well defined. This fact allows us to preserve the notion "triangular array" also for arrays generating sequences of extremal processes as above. The limit behaviour of extremal processes generated by triangular arrays is studied e.g. in [6], [14], [13], [5].

In this paper we treat a particular case of triangular array with $X_{n k}=\xi_{n}^{-1} \circ X_{k}$ and $t_{n k}=\tau_{n}\left(t_{k}\right)$ where the mappings $\zeta_{n}=\left(\tau_{n}, \xi_{n}\right)$ are max-automorphisms of $[0, \infty)^{d+1}$. The point process $\left\{\left(t_{k}, X_{k}\right)\right\}$ is associated with an initial extremal process $X$. Now the partial extremal process $Y_{n}$ in (3) has the form

$$
Y_{n}(t)=\xi_{n}^{-1} \circ X \circ \tau_{n}(t)
$$

Supposing $Y_{n} \Longrightarrow Y$ we are interested in the intrinsic properties of the limit class of extremal processes.

Recall,the max-automorphisms of the form $\zeta(t, x)=\left(\tau(t), \xi_{1}\left(x_{1}\right), \ldots, \xi_{d}\left(x_{d}\right)\right)$ are continuous and strictly increasing in each component. They preserve the max-operation between extremal processes, i.e. $\zeta(X \vee Y)=\zeta(X) \vee \zeta(Y)$, and form a group w.r.t. the composition (cf. [2], [9] ). Since $\tau$ is interpreted as time change and $\xi$ as space change, we call $\zeta$ usually time-space change.

Let $F$ and $G$ be df's on $R^{d}$. We say that $G$ belongs to type(F) if there is a maxautomorphism $L$ of $R^{d}$ such that $G=F \circ L$. 
The basic result in Section 2 states that the limit extremal process for (4) is selfsimilar in the sense that for all $t>0$ there exists a space change $L_{\alpha(t)}$ such that

$$
Y(t) \stackrel{d}{=} L_{\alpha(t)} \circ Y(1),
$$

where $\alpha:(0, \infty) \leftrightarrow(0, \infty)$ is strictly increasing.

The study of the self-similar stochastic processes was initiated by Lamperti (1962). Self-similar extremal processes in a different framework (without the assumption of independence of the max-increments and under use of affine normalization) are investigated in $[8]$.

Equation (5) may be interpreted also as follows :

"All univariate marginals $G_{t}, t>0$, of a self-similar extremal process $Y(t)$ are of the same type."

Under the assumptions of Section 2 it is shown that this type is max-selfdecomposable. The analogous result for self-similar processes with additive increments was proved by Sato [12] already in 1991.

In Section 3 we assume additionally that the initial process $X$ in (4) has homogeneous max-increments. Then the limit class SSHI of self-similar extremal processes with homogeneous max-increments coincides with the intersection of the max-stable extremal processes and the so called (cf. [11]) G-extremal processes. The max-stable extremal processes are studied also in e.g. [3], [5], [6], [8], [11].

Above we have defined extremal processes on the time-state space $[0, \infty) \times[0, \infty)^{d}$. In the same way one defines extremal processes on $(-\infty, \infty) \times[-\infty, \infty)^{d}$ (by allowing mass at $-\vec{\infty}$, cf. [9]) or on $[0,1] \times[0,1]^{d}$, or on any other space homeomorphic to them.

\section{Self-similar extremal processes as limiting}

We start with an extremal process $X:[0, \infty) \rightarrow[0, \infty)^{d}$ with lower curve $C_{X}$, df $f$, and let $N=\left\{\left(t_{k}, X_{k}\right): k \leq 0\right\}$ be the point process generating $X$ by

$$
X(t)=C_{X}(t) \vee \max \left\{X_{k}: t_{k} \leq t\right\}
$$

Here $X_{k}, k \geq 0$, are independent rv's in $[0, \infty)^{d}$ and the sequence $0=t_{0}<t_{1}<\ldots$ of deterministic time points increases to $\infty$. We assume that there exists a sequence $\zeta_{n}=\left(\tau_{n}, \xi_{n}\right)$ of max-automorphisms of $[0, \infty)^{d+1}$ such that the sequence of extremal processes

$$
\begin{aligned}
Y_{n}(t) & =\xi_{n}^{-1} \circ X \circ \tau_{n}(t) \\
& =C_{n}(t) \vee \max \left\{\xi_{n}^{-1} \circ X_{k}: t_{k} \leq \tau_{n}(t)\right\}
\end{aligned}
$$

is convergent weakly in law to a non-degenerate extremal process $Y, Y_{n} \Longrightarrow Y$, with lower curve $C_{Y}$ and df $g$, i.e.

$$
f_{n}(t, x):=f\left(\tau_{n}(t), \xi_{n}(x)\right) \stackrel{w}{\rightarrow} g(t, x)
$$


or briefly

$$
f_{n}=f \circ \zeta_{n} \stackrel{w}{\rightarrow} g
$$

(Under degenerate extremal process we understand here a deterministic one.) The lower curve of $Y_{n}$ is $C_{n}(t)=\xi_{n}^{-1} \circ C_{X}\left(\tau_{n}(t)\right), t \geq 0$. The point process $N_{n}$ in (6) with points

$$
\left\{\left(t_{n k}, X_{n k}\right): k \geq 0\right\}, \quad t_{n k}=\tau_{n}^{-1}\left(t_{k}\right), \quad X_{n k}=\xi_{n}^{-1} \circ X_{k}
$$

form a triangular array of row-wise independent rv's $X_{n k}$ in $[0, \infty)^{d}$. We assume that the max-increments $U_{n}(s, t)$ of $Y_{n}, \quad U_{n}(s, t)=\max \left\{X_{n k}: s<t_{n k} \leq t\right\}, \quad 0 \leq s<t$, are asymptotically negligible in the sense that they obey the following condition

$$
\max _{\left\{k: s<t_{n k} \leq t\right\}} P\left(X_{n k} \in[\overrightarrow{0}, x)^{c}\right) \rightarrow 0, \quad n \rightarrow \infty
$$

for $(t, x) \in A_{Y}$ where the set $A_{Y}$ is determined by its instant sections $A_{Y}^{t}=\left[C_{Y}(t), \vec{\infty}\right) \backslash$ $\left\{C_{Y}(t)\right\}$. As known, in this case the limit extremal process $Y$ is max-id. (The class of the multivariate max-id extremal processes is discussed e.g. in [4] .) Consequently, the df $g$ of the limit extremal process $Y$ is positive on the open set $\operatorname{int} A_{Y}$ above the lower curve $C_{Y}$, hence the family of the max-increments is uniquely determined (cf.[4] )

We are interested in characterizing the class max $-L$ of the possible limit extremal processes for sequences of type (6) or equivalently, the class of the limit df's in (7), under the (AN)-condition.

By (7) for $n$ large enough $\zeta_{n}:\{0<g<1\} \rightarrow\{0<f<1\}$. As a coordinate-wise mapping $\zeta_{n}$ acts on rectangles in $(0, \infty)^{d+1}$. The smallest rectangle $S$ containing the set $\{0<g<1\}$ we call max-support of $g$. Denote $q:=\inf S, w:=\sup S$ and the interiour of $S$ by int $S$. For a sake of simplicity we assume $q=\overrightarrow{0}$, so $C_{Y}(0)=\overrightarrow{0}$, and $w=\vec{\infty}$. Hence $Y:[0, \infty) \rightarrow[0, \infty)^{d}$ Further, ad hock we assume $\zeta_{n}$ increasing in $n$ for normalizing increasing maxima.

To characterize the class max-L using general max-automorphisms as above is a difficult problem for which the necessary theoretical background seems to be not prepared yet (e.g. the convergence to type theorem does not hold in its classical form , cf.[15]). Here we tackle the study of the class $\max -\mathrm{L}(\mathcal{R})$ under the use of regular norming sequences $\left\{\zeta_{n}\right\}$.

Definition: A sequence $\left\{\zeta_{n}\right\}$ of time-space changes is refered to as regular on an increasing subset $B \subseteq[0, \infty)^{d+1}$ (in the sense that $z_{1} \in B$ and $z_{2}>z_{1}$ imply $z_{2} \in B$ ) if for each $\alpha \in(0,1]$ there is a time-space change $\eta_{\alpha}$ such that for $m_{n} \sim \alpha n$ and $n \rightarrow \infty$

$$
\zeta_{n}^{-1} \circ \zeta_{m_{n}}(t, x) \rightarrow \eta_{\alpha}(t, x), \quad(t, x) \in B .
$$

In addition, the correspondence $\alpha \leftrightarrow \eta_{\alpha}$ is one-to-one.

So, we assume that the norming sequence $\zeta_{n}$ in (7) is regular on the max-support $S$ of the limit df $g$. By virtue of (9), the family $\left\{\eta_{\alpha}: \alpha \in(0,1]\right\}$ can be embedded in a one-parameter group $\left\{\eta_{\alpha}: \alpha \in(0, \infty)\right\}$, with

$$
\eta_{\alpha}^{-1}=\eta_{\alpha^{-1}}, \quad \eta_{\alpha} \circ \eta_{\beta}=\eta_{\alpha \beta}, \quad \eta_{1}=\mathrm{id} .
$$


(Here id is the identical mapping.)

Now, for $m_{n}<n, \quad m_{n} \sim n \alpha$ where $\alpha \in(0,1), \quad t>0$ and $I_{n}(t):=\left\{k: \tau_{m_{n}}(t)<\right.$ $\left.t_{k} \leq \tau_{n}(t)\right\}$ let us decompose the extremal process $Y_{n}$ in (6) as

$$
\begin{aligned}
Y_{n}(t) & =\xi_{n}^{-1} \circ X \circ \tau_{n}(t) \\
& =\xi_{n}^{-1} \circ X \circ \tau_{m_{n}}(t) \vee \max \left\{\xi_{n}^{-1} \circ X_{k}: k \in I_{n}(t)\right\}
\end{aligned}
$$

Substituting here

$$
Z_{n, m_{n}}(t):=C_{n}(t) \vee \max \left\{\xi_{n}^{-1} \circ X_{k}: k \in I_{n}(t)\right\}
$$

we can express $Y_{n}(t)$ in two equivalent forms

$$
\begin{aligned}
Y_{n}(t) & =Y_{n}\left(\tau_{n}^{-1} \circ \tau_{m_{n}}(t)\right) \vee Z_{n, m_{n}}(t) \\
& =\left(\xi_{n}^{-1} \circ \xi_{m_{n}}\right) \circ Y_{m_{n}}(t) \vee Z_{n, m_{n}}(t) .
\end{aligned}
$$

Transition to weak limit (along a subsequence if necessary) and the regularity condition (9) with $\eta_{\alpha}:=\left(\sigma_{\alpha}, L_{\alpha}\right), \quad \alpha \in(0,1)$ supply two expressions of the limit extremal process

$$
\begin{aligned}
& Y \stackrel{d}{=} \quad Y \circ \sigma_{\alpha} \vee Z_{\alpha} \\
& \stackrel{d}{=} L_{\alpha} \circ Y \vee Z_{\alpha}
\end{aligned}
$$

Here $Z_{n, m_{n}} \Rightarrow Z_{\alpha}$. Equivalently, the df $g$ of $Y$ satisfies two functional equations

$$
g(t, x)=g\left(\sigma_{\alpha}(t), x\right) \cdot g_{\alpha}(t, x)=g\left(t, \mathbf{L}_{\alpha}^{-1}(x)\right) \cdot g_{\alpha}(t, x)
$$

Here $g_{\alpha}$ is df of the extremal process $Z_{\alpha}$.

So, both expressions of $\xi_{n}^{-1} \circ X \circ \tau_{m_{n}}$ lead to the following characterization of the class $\max -L(\mathcal{R})$ :

$$
Y \circ \sigma_{\alpha} \stackrel{d}{=} L_{\alpha} \circ Y
$$

or

$$
g\left(\sigma_{\alpha}(t), x\right)=g\left(t, L_{\alpha}^{-1}(x)\right)
$$

Below we gather the properties intrinsic for this class of extremal processes.

Definition: An extremal process $Y$ is refered to as self-similar w.r.t. a one-parameter group $\eta_{\alpha}=\left(\sigma_{\alpha}, L_{\alpha}\right)$ of time-space changes if it satisfies equation (13) for all $\alpha \in(0, \infty)$.

From this point of view above we have proved

Proposition 1 The limit extremal process $Y$ is self-similar. 
The family $\left\{\eta_{\alpha}\right\}$ is defined by (9) for $\alpha \in(0, \infty)$. For $\alpha \rightarrow 0$ and $\alpha \rightarrow \infty$ we impose the following natural boundary conditions on $\eta_{\alpha}(t, x)=\left(\sigma_{\alpha}(t), L_{\alpha}^{(1)}\left(x_{1}\right), \ldots, L_{\alpha}^{(d)}\left(x_{d}\right)\right)$ : $(B C)$

$$
\begin{aligned}
\sigma_{\alpha}(t) \rightarrow 0 \quad \text { for } \quad \alpha \rightarrow 0, \quad \sigma_{\alpha}(t) \rightarrow \infty \quad \text { for } \quad \alpha \rightarrow \infty, \\
L_{\alpha}^{(i)}\left(x_{i}\right) \rightarrow 0 \quad \text { for } \quad \alpha \rightarrow 0, \quad L_{\alpha}^{(i)}\left(x_{i}\right) \rightarrow \infty \text { for } \alpha \rightarrow \infty,
\end{aligned}
$$

where 0 and $\infty$ are fixed points of $\sigma_{\alpha}$ and of $L_{\alpha}^{(i)}, i=1 \ldots d$, and $(t, x) \in S$.

Lemma 1 The one-to-one correspondence $\alpha \leftrightarrow \eta_{\alpha}, \alpha \in(0, \infty)$ is strictly increasing, hence continuous.

Indeed, let us assume that $\eta_{\alpha_{1}} \geq \eta_{\alpha_{2}}$ for $\alpha_{1}<\alpha_{2}$. Then $\eta_{r}(z) \geq z$ where $r=\frac{\alpha_{1}}{\alpha_{2}}<1$ and consequently $\eta_{r^{n}}(z) \geq z \quad \forall n>1$ what violates $(\mathrm{BC})$.

Hence, $\left\{\eta_{\alpha}: \alpha \in(0, \infty)\right\}$ is a continuous one-parameter group, briefly c.o.g. Now put $t=1$ and $\sigma_{\alpha}(1)=s$ in (13) and observe that

$$
Y(s) \stackrel{d}{=} L_{\alpha(s)} \circ Y(1)
$$

where $\alpha(s)$ is a solution of $\sigma_{\alpha}(1)=s$. Moreover, this solution is unique, because of lemma 1.

Denote by $G_{s}()=.g(s,$.$) the df of the univariate process marginals. We have state$ Proposition 2 For every $s>0 \quad G_{s} \in$ type $\left(G_{1}\right)$. Furthermore, for each pair $s, t>0$

$$
Y(s) \stackrel{d}{=} L_{\alpha(s, t)} \circ Y(t)
$$

where $\alpha(s, t)=\frac{\alpha(s)}{\alpha(t)}$.

One of the consequences of (2.9) is the following property:

Proposition 3 The limit extremal process $Y$ is stochastically continuous at all $t \geq 0$ and $Y(0)=0 \quad$ a.s.

Proof. Let $s_{n} \uparrow t, \quad t>0$. Then for $x$, continuity point of $g(t, x)$ we have

$$
g(t-0, x)=\lim g\left(s_{n}, x\right)=\lim g\left(t, L_{\frac{\alpha(t)}{\alpha\left(s_{n}\right)}}(x)\right)=g(t, x), \quad n \rightarrow \infty
$$

since $x_{n}:=L_{\frac{\alpha(t)}{\alpha\left(s_{n}\right)}}(x) \downarrow x$ for $n \rightarrow \infty$. Besides, for $\alpha \rightarrow 0$

$$
g(0, x)=\lim g\left(\sigma_{\alpha}(1), x\right)=\lim g\left(1, L_{\alpha^{-1}}(x)\right)=g(1, \vec{\infty}) .
$$

(Here we have used the lower semicontinuity of $g$.) Hence

$$
P(Y(0)<x)=P(Y(1)<\vec{\infty}) \quad \text { for } \quad x>C_{Y}(0)=\overrightarrow{0} .
$$

Obviously $Y(0)=0$ a.s., since $G_{1}$ does not allow mass at the upper boundary, i.e. $P(Y(1)<\vec{\infty})=1$, and $Y$ is stochastically continuous at all $t \geq 0$.

Stochastic continuity of the extremal process does not imply continuity of the lower curve. However, there is another nice consequence of (2.9) : 
Proposition 4 The lower curve $C_{Y}$ is continuous.

Indeed, $L_{\alpha(t)}:\left(C_{Y}(1), \vec{\infty}\right) \leftrightarrow\left(C_{Y}(t), \vec{\infty}\right)$. So, all lower vertex $C_{Y}(t)$ of $G_{t}$ lie on the same orbit of $L_{\alpha(t)}$ through $C_{Y}(1)$.

The limit extremal process $Y$ is max-id. By theorem 1 in [4], $Y$ is Poisson, i.e. it is generated by a Poisson point process $N$. Now proposition 2 and decomposition theorem 2 determine $N$ (but not uniquely, because of the phenomenon blotting : two point processes $N_{1}$ and $N_{2}$ on $\left[\overrightarrow{0}, C_{Y}\right]^{c}$ that coincide on the set $A_{Y}$ above the lower curve $C_{Y}$, but differ on $\left[\overrightarrow{0}, C_{Y}\right]^{c} \backslash A_{Y}$, generate the same extremal process $Y$ ).

Proposition 5 The associated point process $N$ to the limit extremal process $Y$ is Poisson. It is sum of a Poisson point process $N^{\prime}$ which mean measure does not charge instant spaces $S_{t}:=\{t\} \times[0, \infty)^{d}, \quad t>0$, and a 0-1 point process $N_{0}=\{(0, Y(0))\}$ where $Y(0)$ is $\max -i d$.

Let us come back to the decomposition (12). The extremal process $Z_{\alpha}$ is max-id , too, since limiting for triangular array with (AN)-condition. It has the same lower curve $C_{Y}$. Now the functional equation

$$
g(t, x)=g\left(t, L_{\alpha}^{-1}(x) \cdot g_{\alpha}(t, x)\right.
$$

can be interpreted as follows.

Proposition 6 For all $t>0$ the univariate marginals $G_{t}()=.g(t,$.$) of the limit extremal$ process $Y$ are max-selfdecomposable w.r.t. the semigroup $\left\{L_{\alpha}^{-1}: \alpha \in(0,1]\right\}$ of space changes, i.e.

$$
G_{t}(x)=G_{t}\left(L_{\alpha}^{-1}(x)\right) \cdot G_{t, \alpha}(x)
$$

The component $G_{t, \alpha}(x)=g_{\alpha}(t, x)$ is max-id. The max-selfdecomposability w.r.t. a one-parameter semigroup of max-automorphisms of $R^{d}$ is discussed in [9]. Such df G is continuous everywhere except may be on the boundary of the support. One consequence of (15) is the inequality $x<L_{\alpha}^{-1}(x)$, i.e. the mapping $L_{\alpha}$ is contracting for $\alpha \in(0,1)$. Analogously, from the first equation in $(2.7 \mathrm{a})$ we conclude that $\sigma_{\alpha}(t)$ for $\alpha \in(0,1)$.

Let us denote the invariant (or symmetric) group of $g$ by

$$
\operatorname{Inv}(g):=\left\{\text { time-space changes } \eta \text { of }[0, \infty)^{d+1}: g \circ \eta=g\right\}
$$

The force of characteristic equation (2.8a) written also as

$$
g(t, x)=g\left(\sigma_{\alpha}(t), L_{\alpha}(x)\right)
$$

is stressed by the next statement.

Proposition $7 \operatorname{Inv}(g)$ contains a c.o.g. $\left\{\eta_{\alpha}: \alpha \in(0, \infty)\right\}$. 
As known, the compactness of $\operatorname{Inv}(\mathrm{g})$ is necessary and sufficient for the application of the Convergence to Type Theorem in limit relation (7).

We have already observed that every extremal process $Y \in \max -L(\mathcal{R})$ is self-similar and all its increments $U(s, t), \quad 0 \leq s<t$, are max-id (since Y is max-id). The converse statement is also true : any self-similar extremal process (with max-id increments) is limiting for a sequence $Y_{n}=\xi_{n}^{-1} \circ X \circ \tau_{n}$ where the norming sequence is regular and the max-increments of $Y_{n}$ are asymptotically negligible. To see this we need the following two statements.

Lemma 2 Let $Y_{n}, \quad n \geq 0$, be extremal processes with df's $f_{n}$. If $Y_{n} \Longrightarrow Y_{0}$ and $Y_{0}$ is stochastically continuous, then the sequence $Y_{n}$ is asymptotically continuous, i.e. the sequence of $d f$ 's $f_{n}$ satisfies the condition

$$
\max _{0<t \leq c}\left[f_{n}(t-0)-f_{n}(t)\right] \rightarrow 0, \quad n \rightarrow \infty
$$

for all $c>0$.

Proof. Indeed, $Y_{0}$ stochastically continuous and $Y_{n} \Longrightarrow Y_{0}$ imply

$$
f_{n}(t-0)-f_{n}(t) \rightarrow f_{0}(t-0)-f_{0}(t)=0 \quad \forall t>0, \quad n \rightarrow \infty .
$$

Both conditions (AC) and (AN) are closely related as the following theorem states.

Theorem 3 Assume $Y_{n} \Longrightarrow Y_{0}$. If the sequence $Y_{n}$ is asymptotically continuous then it has asymptotically negligible max-increments $U_{n}((s, t])$ for $0 \leq s<t$. The converse holds under the additional continuity assumption on the limit process: $Y_{0}(t-0) \geq C_{0}(t)$ a.s. for $t>0$. This condition is automatically fulfield if the lower curve $C_{0}$ of $Y_{0}$ is continuous .

Proof. Denote the df of $U_{n}$ by $H_{n}$. The max-increments $U_{n}((s, t]), \quad 0 \leq s<t$, are asymptotically negligible iff

$$
H_{n, t}(x)=P\left(U_{n}(t) \in[\overrightarrow{0}, x)\right) \rightarrow 1, \quad n \rightarrow \infty,
$$

for $t>0$ and $\forall x>C_{0}(t)$. On the other hand, by the decomposition theorem

$$
Y_{n}(t)=Y_{n}(t-0) \vee U_{n}(t) .
$$

Thus, condition (AN)' means that

$$
H_{n, t}(x)=\frac{f_{n}(t, x)}{f_{n}(t-0, x)} \rightarrow 1, \quad n \rightarrow \infty
$$

for $t>0$ and $x>C_{0}(t)$. 
The sequence $Y_{n}$ is asymptotically continuous iff the asymptotic relation (2.11) holds for all $t>0$ and $x \in[0, \infty)^{d}$. Obviously critical values are $x \in\left(C_{0}(t-0), C_{0}(t)\right)$ for which (2.11) may be not fulfiled. This case is avoided by the additional assumption $Y_{0}(t-0) \geq C_{0}(t)$ a.s.

Note, $Y_{n}$ asymptotically continuous and $Y_{n} \Longrightarrow Y_{0}$ does not imply $U_{n}(0) \rightarrow C_{0}(0)$. Now we can prove the main statement of this section.

Theorem 4 The class $\max -L(\mathcal{R})$ coincides with the class of extremal processes which are self-similar w.r.t. a c.o.g. $\left\{\eta_{\alpha}: \alpha \in(0, \infty)\right\}$ of time-space changes satisfying the $(B C)$-condition.

Proof. We have still to show that if $Y$ is self-similar, then $Y \in \max -L(\mathcal{R})$. The self-similarity condition implies that the extremal process $Y$ is stochastically continuous at $t>0$, its lower curve $C_{Y}$ is continuous and its $\mathrm{df} g$ satisfies the functional equation $g\left(\sigma_{\alpha}(t), L_{\alpha}(x)\right)=g(t, x)$. Let $N=\left\{\left(t_{k}, Y_{k}\right): k \geq 0\right\}$ be the point process generating $Y$ by

$$
Y(t)=C_{Y}(t) \vee \max \left\{Y_{k}: 0 \leq t_{k} \leq t\right\} .
$$

Here $t_{0}=0$ and $Y_{0}=Y(0)=0$ a.s. Define $t_{n k}=\sigma_{n}^{-1}\left(t_{k}\right), \quad X_{n k}=L_{n}^{-1} \circ Y_{k}$ for $\alpha=n, \quad n \geq 1$, and observe that $t_{n 0}=0, \quad X_{n 0} \stackrel{d}{=} Y(0)$, so the sequence

$$
Y_{n}(t):=C_{n}(t) \vee \max \left\{X_{n k}: 0<t_{n k} \leq t\right\}=L_{n}^{-1} \circ Y \circ \sigma_{n}(t) \stackrel{d}{=} Y(t)
$$

is trivially convergent. Here the norming sequence $\eta_{n}$ is regular.

By lemma 2 and theorem 3 the max-increments of $Y_{n}$ over intervals $(s, t], \quad 0 \leq s<t$ , are asymptotically negligible. Hence, $Y$ belongs to the class $\max -L(\mathcal{R})$.

The class $\max -L(\mathcal{R})$ we have determined as the class of all weak limits for sequences (2.1) where $\zeta_{n}$ is a regular norming sequence and $X$ is a non-degenerate extremal process, such that triangular array (2.3) obey the (AN)-condition. Then the limit extremal process $Y$ is stochastically continuous and has continuous lower curve. In this special case both kinds of convergence, $Y_{n} \Longrightarrow Y$ weakly and $Y_{n} \Longrightarrow Y$ in $D([0, \infty))$, are equvalent provided we assume convergence of the initial values $Y_{n}(0) \Longrightarrow Y(0)$.

As a matter of fact, both conditions (2.5) and (BC) determine the analytical form of the time-space changes $\eta_{\alpha}$ on $\mathrm{S}$ as the following lemma claims.

Lemma 3 The continuous one-parameter group $\left\{\eta_{\alpha}: \alpha \in(0, \infty)\right\}$ of time-space changes of $[0, \infty)^{d+1}, \quad \eta_{\alpha}: S \leftrightarrow S$, satisfying the boundary conditions $(B C)$, can be expressed on $S$ in the form :

$$
\eta_{\alpha}(z)=h^{-1}(h(z)+\text { e.c. } \log \alpha)
$$

where $e=(1, \ldots, 1) \in R^{d+1}, \quad c>0$, and $h: S \leftrightarrow(-\infty, \infty)^{d+1}$ is continuous and strictly increasing coordinatewise mapping. 
The proof of this lemma is a modification of theorem 20 in [1]. Expression (2.12) means that there exists a time-space change $h: S \leftrightarrow R^{d+1}$ so that, in the new coordinates $z^{\prime}=h(z)$, the one-parameter group $\eta_{\alpha}^{\prime}=h \circ \eta_{\alpha}$ is a simple translation along the diagonal in $R^{d+1}$, i.e.

$$
\eta_{\alpha}^{\prime}(z)=z^{\prime}+e \cdot \theta(\alpha)
$$

with $\theta(\alpha)=c \log \alpha \in(-\infty, \infty)$. Denote the translation group along the diagonal by $D_{r}(z):=z+e r, z \in R^{d+1}, r \in R^{1}$. Note, $D_{r} . D_{s}=D_{d+s}, \quad D_{0}=i d, \quad D_{r}^{-1}=D_{-r}$.

Definition: An extremal process $Y:(-\infty, \infty) \rightarrow[-\infty, \infty)^{d}$ with df $\mathrm{g}$ is called diagonal if for all $r \in R^{1} \quad g \circ D_{r}=g$.

In other words, diagonal means selfsimilar w.r.t. the translation group.

Since $g \circ \eta_{\alpha}(z)=g \circ h^{-1}\left(z^{\prime}+e \theta\right)$, in fact theorem 4 claims

"The class $\max -L(\mathcal{R})$ consists of all extremal processes $Y$ related by a time-space change $h: S \leftrightarrow R^{d+1}$ to a diagonal process $M$,i.e.

$$
Y \stackrel{d}{=} h^{-1} \circ M \quad "
$$

\section{Self-similar extremal processes with homogeneous max-increments}

Here we consider the same stochastic model as in Section 2 with one additional condition : the initial extremal process $X$ has homogeneous max-increments, i.e. the associated increments process

$$
U_{X}(s, t)=C_{X}(t) \vee \max \left\{X_{k}: s<t_{k} \leq t\right\}, \quad 0 \leq s<t,
$$

satisfies

$$
U_{X}(s, t) \stackrel{d}{=} U_{X}(0, t-s) .
$$

Then the limit extremal process $Y$ (besides that it is self-similar) has some additional properties. Our next goal is to state them.

Consider the partial extremal process $Y_{n}(t)=\xi_{n}^{-1} \circ X \circ \tau_{n}(t)$. For arbitrary $s, 0 \leq$ $s<t$, let $m_{n}=m_{n}(s)$ be a subsequence of integers such that $\tau_{n}^{-1} \circ \tau_{m_{n}}(t) \rightarrow s>0$. Then the decomposition

$$
Y_{n}(t)=Y_{n}\left(\tau_{n}^{-1} \circ \tau_{m_{n}}(t)\right) \vee \max \left\{\xi_{n}^{-1} \circ X_{k}: \tau_{n}^{-1} \circ \tau_{m_{n}}(t)<\tau_{n}^{-1}\left(t_{k}\right) \leq t\right\}
$$

supplies the following equation for the limiting extremal process $Y$

$$
Y(t) \stackrel{d}{=} Y(s) \vee Y(t-s)
$$

On the other hand

$$
Y(t)=Y(s) \vee U_{Y}(s, t) \quad a . s .
$$


by the structure theorem. The family $\left\{U_{Y}(s, t)\right\}, U_{Y}(s, t) \geq C_{Y}(t)$ a.s., of the maxincrements of $Y$ is uniquely determined as $Y$ is max-id. Let $H_{s, t}$ be the df of $U_{Y}(s, t)$. Comparing the last two equations for $Y(t)$ we observe

$$
U_{Y}(s, t) \stackrel{d}{=} C_{Y}(t) \vee Y(t-s)
$$

or equivalently

$$
H_{s, t}(x)=G_{t}(x) / G_{s}(x)=G_{t-s}(x)
$$

The df $g$ of the limit process $Y$ satisfies the following functional equation for $x>$ $C_{Y}(t)$ :

$$
g(t, x)=g(s, x) \cdot g(t-s, x) \quad, \quad s<t .
$$

The solution of 19 is well-known, namely

$$
g(t, x)=G^{t}(x)
$$

where $G(x)=P(Y(1)<x)$ and $\mathrm{G}$ is a max-id $\mathrm{df}$ on $[0, \infty)^{d}$. Thus

$$
P\left(U_{Y}(s, t)<x\right)=G^{t-s}(x)
$$

Now the self-similarity of $Y$, namely $Y(t)=L_{\alpha(t)} \circ Y(1)$ implies

$$
G^{t}(x)=G\left(\mathbf{L}_{t}^{-1}(x)\right)
$$

for all $t>0$, where $\left\{\mathbf{L}_{t}:=L_{\alpha(t)}, \quad t>0\right\}$ is a c.o.g. Functional equation (3.4) is characteristic for the class of max-stable df's (cf. [9]). Thus we have:

Proposition 8 All univariate marginals of $Y$ belong to the same type and this type is max-stable w.r.t. the one-parameter group $\left\{\mathbf{L}_{t}, t>0\right\}$ of space changes.

Corollaries: 1) $P(Y(0)=0)=1$.

Indeed, $\mathbf{L}_{t} \rightarrow C_{Y}(0)$ for $t \rightarrow 0$ and $x \in\{0<G<1\}$, and we have assumed $C_{Y}(0)=0$. The LHS of (3.4) equals 1 for $t=0$.

2) $Y(a s) \stackrel{d}{=} \mathbf{L}_{a} \circ Y(s), \forall a>0$.

3) $Y$ is stochastically continuous for all $t \geq 0$.

In view of (3.1) and (3.3) we conclude

$$
H_{s, t}(x)=\frac{g(t-s, x)}{g(0, x)}=H_{0, t-s}(x) .
$$

Thus, we state

Proposition 9 The limit extremal process has homogeneous max-increments.

Hence,normalization with regular sequences and transition to weak limit preserve the homogenity property of the initial process $X$. 
Proposition 10 The finite dimensional distributions (fdd) of $Y$ are of the form

$$
\begin{aligned}
& \qquad P\left(Y\left(t_{1}\right)<x_{1}, \ldots, Y\left(t_{k}\right)<x_{k}\right)=G^{t_{1}}\left(x_{1}\right) \cdot G^{t_{2}-t_{1}}\left(x_{2}\right) \ldots G^{t_{k}-t_{k-1}}\left(x_{k}\right) \\
& \text { for } 0<t_{1}<\ldots<t_{k}, \quad x_{1}<\ldots<x_{k} \text { and } G(x)=P(Y(1)<x)
\end{aligned}
$$

In [11] extremal processes with these fdd are called G-extremal processes. We denote their class by $\mathcal{R}$. ( Here $\mathcal{R}$ stays for Resnick and Rubinovich.) The extremal processes of the class $\mathcal{R}$ are stochastically continuous processes starting at the origin with independent and homogeneous max-increments, hence we may consider them Levy processes in the max-framework.

Note, the type of an extremal process is determined by the type of its max-increments. In general the type of the univariate marginals of an extremal process does not determine the type of the process itself. E.g. given $G_{t}(x)=P(Y(t)<x)$ is max-id $\forall t>0$, we can not claim that the quotient

$$
H_{s, t}(x)=P\left(U_{Y}(s, t)<x\right)=\frac{G_{t}(x)}{G_{s}(x)}
$$

(hence the process $Y$ ) is max-id, too. (Recall, a max-id df may have indecomposable components, cf [10].) But in our case, equations (3.3) and (3.4) mean that the increments process is max-stable, too.

Proposition 11 The type of the limit extremal process $Y$ is uniquely determined by the type of the univariate marginals, namely $Y$ is max-stable (briefly, $Y \in M S$ ).

The last means : for all integers $\mathrm{n}$ there exist iid extremal processes $Y_{1}, \ldots, Y_{n}$,copies of $Y$, and space change $L_{n}$ such that

$$
Y \stackrel{d}{=} L_{n}^{-1}\left(Y_{1} \vee \ldots \vee Y_{n}\right)
$$

(cf.[3] and [5]).

Consider functional equation (3.4) ones more. Another consequence of it is the next property.

Proposition 12 The lower curve $C_{Y}$ of the limit extremal process $Y$ is constant, i.e. $C_{Y}(t) \equiv C_{Y}(1)=\inf \{G>0\}$.

Denote the class of the possible limit extremal processes for triangular arrays described in this Section by SSHI. We have observed that every $Y \in S S H I$ is a selfsimilar extremal process with homogeneous max-id increments. Propositions 9 and 3.4 stress the inclusion $S S H I \subset M S \cap \mathcal{R}$.

The converse observation is also true. Indeed, let $Y$ be a max-stable extremal process with homogeneous max-increments and df $g$. Hence $\forall t>0 \quad g(t, x)=G^{t}(x)=$ $G\left(\mathbf{L}_{t}^{-1}(x)\right)$ where $\mathrm{G}$ is the $\mathrm{df}$ of the $\mathrm{rv} Y(1)$ and $\inf \{G>0\}=: q \geq 0$. Define a rv 
$X \stackrel{d}{=} Y(1)$ and let $X_{1}, \ldots, X_{n}$ be iid copies of $X$. Put $t_{n k}:=\frac{k}{n}, \quad X_{n k}:=\mathbf{L}_{n}^{-1} \circ X_{k}$. Then the triangular array $\left\{\left(t_{n k}, X_{n k}\right)\right\}$ generates a sequence of extremal processes $Y_{n}$,

$$
Y_{n}(t)=q \vee \max \left\{\mathbf{L}_{n}^{-1} \circ X_{k}: k \leq n t\right\},
$$

which is convergent weakly in law to the initial extremal process $Y$, namely

$$
P\left(Y_{n}(t)<x\right)=G^{[n t]}\left(\mathbf{L}_{n}(x)\right) \sim G^{t}(x)=P(Y(t)<x)
$$

The partial extremal process $Y_{n}$ can be expressed also as

$$
Y_{n}(t)=\mathbf{L}_{n}^{-1} \circ X \circ \tau_{n}(t)
$$

where $\tau_{n}(t)=n t$ and $X(t):=q \vee \max \left\{X_{k}: k \leq t\right\}$. The extremal process $X$ has homogeneous max-increments $U_{X}(s, t)$ (since $X_{k}$ are iidrv) and its df $f$ is

$$
f(t, x)=P(X(t)<x)=G^{[t]}(x) .
$$

Obviously, the norming sequence $\zeta_{n}=\left(\tau_{n}, \mathbf{L}_{n}\right)$ is regular. Further, the stochastic continuity of the limit extremal process $Y$ in (3.5) garantees the asymptotic continuity of the sequence $Y_{n}$ which implies the (AN)-condition for the max-increments of $Y_{n}$. Consequently, the process $Y$ belongs to the class SSHI and so we have established the following property, characteristic for the limit class.

Proposition 13 The class SSHI coincides with the class of all self-similar extremal processes with homogeneous max-increments. So, SSHI $=\mathcal{R} \cap M S$.

Example. Let $Y$ be an extremal process with df

$$
g(t, x)= \begin{cases}0 & \text { for } \quad x \leq 0 \\ \exp \left\{-\frac{t}{x^{\gamma}}\right\} & \text { for } \quad x>0, \gamma>0 .\end{cases}
$$

Obviously, $g(t, x)=g\left(\alpha t, \alpha^{H} x\right)$ where $H=\frac{1}{\gamma}$. Thus, $Y$ is self-similar w.r.t. the c.o.g. $\eta_{\alpha}$ with $\sigma_{\alpha}(t)=\alpha t, \quad L_{\alpha}(x)=\alpha^{H} x$. Further, $g_{t}(x)=\left(e^{-x^{-\gamma}}\right)^{t}, \quad g_{1}(x)=\Phi_{\gamma}(x)$, i.e. $Y \in S S H I$.

Acknowledgements. The author gratefully acknowledges a grant from NWO,the Dutch organization for scientific research. She expresses a deep gratitude to A.A.Balkema for introducing her in the nice theory of extremal processes and for very helpful comments.

\section{Bibliography}

[1] J. ACZEL. Some general methods in the theory of functional equations with one variable. New applications of functional equations (in Russian). Uspehi Mathem. Nauk 3(69) (1956), $3-67$. 
[2] A. A. Balkema. Monotone transformations and limit laws. PhD Thesises, Mathem.Centrum - Amsterdam, 1973.

[3] A. A. Balkema, L. De Haan, R. L. Karandikar. Asymptotic distribution of the maximum of $\mathrm{n}$ independent stochastic processes. J. Appl. Prob. 30 (1993), 66-81.

[4] A. A. Balkema, E. I. Pancheva. Decomposition for multivariate extremal processes. Commun. Statist.-Theory Meth., 25(4)(1996), 737-758.

[5] E. Gine, M. G. Hahn, P, Vatan. Max-infinitely divisible and max-stable sample continuous processes. Probab. Th. Rel. Fields 87 (1990), 139-165.

[6] J. Lamperti. On extreme order statistics. Ann. Math. Stat. 35 (1964), 1726-1737.

[7] J. Lamperti. Semi-stable stochastic processes. Trans. Amer. Math. Scc. 104 (1962), 62-78.

[8] G. L. O'Brien, P.J.J.F. Torfs, W. VervaAt. Stationary selfsimilar extremal processes. Probab. Th. Rel. Fields 87 (1990), 97-119.

[9] E. I. PAncheva. Extreme value limit theory with non-linear normalization. In: Extreme value theory and applications, Kluwer Acad.Publ., 1994, 305-318.

[10] E. I. Pancheva. On a problem of a Khinchin-type decomposition theorem for extreme values. Probab. Theory Appl. 39(2) (1994), 395-402.

[11] S. I. Resnick, M. Rubinovich. The structure of extremal processes. Adv. Appl. Prob.5 (1973), 287-307.

[12] K. Sato. Self-similar processes with independent increments. Probab. Theory Rel. Fields 89(3) (1991), 285-300.

[13] R. SERFozo. Functional limit theorems for extreme values of arrays of independent random variables. Ann. Probab., 10(1) (1982), 172-177.

[14] I. Weissman. Multivariate extremal processes generated by independent non-identically distributed randon variables. J. Appl. Prob., 12 (1975), 477-487.

[15] E. I. Pancheva. Convergence of types under monotone mappings. Probab. Theory Appl., 38(4) (1993), 679-684.

Dept.of Probability and Statistics

Institute of Mathematics

Bulgarian Academy of Sciences

Acad. G.Bontchev str., bl. 8

1113 Sofia, Bulgaria

e-mail: pancheva@math.bas.bg 